\title{
Evidence-based pharmacogenetics: Is it possible?
}

\author{
D.A. Sychev ${ }^{\mathrm{a}, \mathrm{b}}$ and E.U. Malova $\mathrm{a}^{\mathrm{a}, \mathrm{b}, *}$ \\ ${ }^{a}$ Russian Medical Academy of Post-Graduate Education (RMAPE), Moscow, Russia \\ ${ }^{\mathrm{b}}$ I.M. Sechenov First Moscow State Medical University, Moscow, Russia
}

*Corresponding author. E-mail: malovapmgmu@gmail.com

\begin{abstract}
BACKGROUND: For improving quality, safety and efficiency of care, health systems perform a paradigm change towards personalized medicine, also referred to as genomic medicine. It uses combined knowledge (genomics, transcriptomics, proteomics, metabolomics) about a person to predict disease susceptibility, disease prognosis or treatment response and thereby to improve the person's health. The last decade has witnessed a steady embrace of personalized medicine by senior government officials, industry leadership and health care providers [1]. On the 12th December of 2013 Russian President Vladimir Putin in his annual address to the Federal Assembly said: "The Ministry of Health and the Russian Academy of Sciences must give priority to fundamental and applied research in medicine, including genomic studies" [2]. A year earlier, in 2012 the Ministry of Health of the Russian Federation, headed by Veronika Skvortsova established the strategy of personalized medicine development in Russia [3]. But still a lot of work is focused on using clinical research findings to aid the delivery of optimum clinical care to patients. Pharmacogenetic testing (using genetic information to guide drug therapy) is an actively developing field of personalized medicine and its current state indicates that it can be usefully introduced into clinical practice in the nearest future. In Russia pharmacogenetic testing is already used for personalizing prescription of certain drugs [4].
\end{abstract}

OBJECTIVE: To assess the extent of genetic testing use for improving use of medicines.

METHODS: PubMed and E-Library searches for the period of 2004-2015.

RESULTS: The number of publications retrieved in PubMed search for the term "pharmacogenetics" for 2004 year was 538 and was more than 15500 publications for 2015. 800 Russian-language publications in total were retrieved using a domestic scientific database E-Library search for the term "pharmacogenetics" for 2015 year. The sharp rise in the number of publications (including Russia) reflects growing interest not only among scientists, but also among practitioners. However evidence that is actually available on some key topics may not be of sufficiently high quality to support confident conclusions. As a rule, retrospective cohort studies, also known as historical cohort studies, are carried out. The number of randomized, prospective studies is not large, though in recent years, there has been an increase in their number. However, surrogate outcomes are commonly used in the mentioned studies as trial end points. The main reason for this is the lack of sponsorship. Quite often studies are not interesting for pharmaceutical companies and are carried out within the confines of the small grants. Nevertheless, systematic reviews and meta-analyses of some pharmacogenetic tests provide the high 
level of evidence (pharmacogenetic testing for clopidogrel, abacavir and antineoplastic drugs) so they appear even in clinical guidelines with the evidence level IIb. It is important to mention that for certain drugs FDA has already approved pharmacogenetic testing [5].

CONCLUSIONS: Evidence is often inconsistent. This leads to the fact that clinical use of pharmacogenetic testing seems to be most appropriate for the management of patients with high risk of adverse drug reactions.

Keywords: Pharmacogenetics, evidence, personalized medicine

\section{Conflict of interest statement: None.}

\section{References}

[1] Blobel B. Translational medicine meets new technologies for enabling personalized care. stud Health Technol Inform. 2013;189:8-23 doi: 10.3233/978-1-61499-268-4-8

[2] V.V. Putin, the annual address to the Federal Assembly [Internet] 2013 Dec 12 [cited 2013 Dec 12 ]. Available from: http://www.kremlin.ru/news/19825

[3] Strategy of personalized medicine development in Russia [Internet] 2012 Dec 10 [cited 2012 Dec 10 ]. Available from: http://medportal.ru/mednovosti/news/2012/12/10/personal

[4] Johnson JA, Cavallari LH. Pharmacogenetics and cardiovascular disease-implications for personalized medicine. Pharmacol Rev. 2013 May 17;65(3):987-1009. doi: 10.1124/pr.112.007252

[5] Table of Pharmacogenomic Biomarkers in Drug Labeling. [Internet] 2015 May 20 [last updated 2015 May 20 ]. Available from: http://www.fda.gov/drugs/scienceresearch/researchareas/pharmacogenetics/ucm083378.htm 